\title{
Paladar o Tuberosidad, ¿Cuál es la Mejor Zona Donadora para un Injerto de Tejido Blando?: Una Revisión Sistemática
}

\author{
Palate or Tuberosity, Which is the Best Donor Site for a Soft tissue graft? \\ A Systematic Review
}

\author{
Edwin Ramos-Pilco ${ }^{1,2}$; Yesica Condori Salinas ${ }^{1,2} \&$ Marco Antonio Alarcón ${ }^{1,3}$
}

\begin{abstract}
RAMOS-PILCO, E.; CONDORI, S. Y. \& ALARCÓN, M. A. Paladar o Tuberosidad, ¿Cuál es la mejor zona donadora para un injerto de tejido blando?: Una revisión sistemática. Int. J. Odontostomat., 14(4):602-609, 2020.

RESUMEN: Comparar mediante una revisión sistemática los resultados clínicos de los procedimientos de cirugía plástica periodontal/periimplantar (CP) con injerto de tejido blando autógeno (ITB) obtenido del área lateral del paladar (ALP) versus del área de la tuberosidad (AT). Se realizó una búsqueda electrónica de ensayos clínicos en la base de datos Medline/Pubmed, Cochrane y las revistas de Periodoncia e Implantes de mayor impacto según la Web of Science, para hallar artículos publicados hasta abril del 2020. Se valoró el riesgo de sesgo de los artículos añadidos según el manual Cochrane Versión 5.1.0 para ensayos clínicos aleatorizados y la escala Newcastle-Ottawa para ensayos clínicos controlados. De una muestra inicial de 930 artículos, cuatro ensayos clínicos (tres aleatorizados) fueron incluidos en el presente estudio, donde se realizaron un total de $87 \mathrm{CP}$ alrededor de piezas e implantes dentales, de las cuales 42 cirugías fueron realizadas con ITB del ALP y 45 cirugías con ITB del AT, se evaluó los resultados desde las 8 semanas hasta los 12 meses. No se hallaron diferencias estadísticamente significativas en los resultados clínicos entre ambos grupos, se mejoró el fenotipo gingival en la zona receptora para el grupo que obtuvo el ITB del AT y el nivel del dolor del sitio donador del AT fue menor en las dos primeras semanas que el sitio donante del ALP. Los estudios incluidos manifestaron un bajo riesgo de sesgo en promedio. Ambas áreas donantes de injerto de tejido blando proporcionan resultados clínicos similares, el injerto del área de la tuberosidad mejora el fenotipo gingival de la zona receptora y reduce el dolor post operatorio en las primeras semanas del sitio donador.
\end{abstract}

PALABRAS CLAVE: implantes dentales, paladar, tejido conectivo, autoinjerto, recesión gingival.

\section{INTRODUCCIÓN}

Los procedimientos de cirugía plástica periodontal/periimplantar (CP) tales como el aumento de tejido queratinizado, recubrimiento de recesiones gingivales, cubrimiento de recesiones de la mucosa periimplantar e incremento de volumen con injerto de tejido blando (ITB) están indicados para establecer la función y la estabilidad de los tejidos alrededor de los dientes, implantes y rebordes edéntulos (Cairo et al., 2008; Thoma et al., 2014). En torno a los implantes dentales, los defectos de tejido blando son hallazgos clínicos comunes, su presencia puede conducir una pérdida ósea crestal, inflamación y recesión tisular (Schwarz et al., 2012; Jung et al., 2017; Hämmerle \& Tarnow, 2018).
El injerto de tejido conectivo autógeno (ITC) es considerado el material de elección (gold standard) para los procedimientos de CP (Thoma et al.; Zhur et al., 2014). EI ITC actúa como un andamio biológico que mejora la estabilidad del colgajo a la superficie radicular, promoviendo un mayor grosor de tejido blando y un ancho de tejido queratinizado al mismo tiempo (Zucchelli \& Mounssif, 2015). En la colocación de implantes tipo I (post extracción) la adición del ITC puede dar resultados superiores con respecto a la altura de la papila y al nivel de la mucosa marginal (Thoma et al.).

El ITC se obtiene de la mucosa palatina existiendo diversas técnicas de recolección que dan como

\footnotetext{
${ }^{1}$ Cirujano Dentista. Especialista en Periodoncia e Implantes, Perú.

2 Periodoncia e Implantología, Facultad de Ciencias de la Salud, Universidad Privada de Tacna, Tacna, Perú.

${ }^{3}$ Departamento Académico de Clínica Estomatológica, Facultad de Estomatología, Universidad Peruana Cayetano Heredia, Perú.
} 
resultado diferentes características del injerto, tanto en tamaño y composición histológica. El área lateral del paladar (ALP) tiene una consistencia flexible, pero puede ser más extensa mientras el área de la tuberosidad (AT) es más densa pero de tamaño limitado (Sanz et al., 2014), además en el ALP el ITC profundo parece no tener el mismo potencial de inducir queratinización como el ITC superficial, que puede deberse a la gran cantidad de tejido adiposo y glandular, tejido que puede actuar como barrera a la difusión plasmática y revascularización durante la primera fase de cicatrización (Sculean et al., 2014).

El objetivo del presente estudio fue comparar los resultados clínicos de los procedimientos de $\mathrm{CP}$ con injerto de tejido blando obtenido del área lateral del paladar versus del área de la Tuberosidad.

\section{MATERIAL Y MÉTODO}

Protocolo y pregunta de investigación. Se desarrolló un protocolo a priori, con la finalidad de contestar la siguiente interrogante: ¿Cuál es la mejor zona dadora de injerto de tejido blando para los procedimientos de cirugía plástica periodontal/periimplantar? Este protocolo cumplió las sugerencias del reporte para revisiones sistemáticas PRISMA (Moher et al., 2009).

\section{Criterios de inclusión de los artículos (PICOS)}

- Población: Pacientes con indicación de cirugía plástica periodontal/periimplantar con injerto de tejido blando autógeno, con salud periodontal y sin compromiso sistémico.

- Intervención: Cirugía plástica periodontal/ periimplantar con ITB del AT.

- Comparación: Cirugía plástica periodontal/ periimplantar con ITB del ALP.

- Resultados: Métodos de medición bidimensionales o tridimensionales a partir de las 8 semanas.

- Diseño de estudio: Ensayos clínicos aleatorizados (ECA) y controlados (ECC)

Criterios de exclusión de los artículos. Estudios que se realicen en pacientes con hábitos nocivos (fumadores, drogas), embarazo, lactancia y menores de 18 años.
Tipos de resultados. El objetivo primario de la presente revisión sistemática fue comparar los resultados clínicos del tratamiento de cirugía plástica periodontal/periimplantar con ITB tomado del área lateral del paladar versus el área de la tuberosidad, empleando métodos de medición bidimensionales o tridimensionales. El objetivo secundario fue comparar los resultados clínicos del fenotipo gingival de las zonas receptoras y el dolor postoperatorio de las zonas dadoras.

Búsqueda electrónica y manual. Se ejecutó una búsqueda global en la base de datos MEDLINE (PubMed) y en la biblioteca Cochrane, para identificar ensayos clínicos hasta abril del 2020. La técnica de búsqueda utilizó las siguientes palabras: ((()((Soft tissue graft) OR Soft tissue grafting) OR Soft tissue augmentation) OR Connective tissue graft) OR subepithelial connective tissue)) AND (((((palate) OR palate lateral) OR palatal area) OR tuberosity) OR tuberosity area), adicionalmente se examinaron las listas de referencia de cualquier artículo potencial.

Se realizó una búsqueda manual de las revistas de mayor impacto en Periodoncia e Implantología oral según la Web of Science: Journal of Clinical Periodontology, Journal of Periodontology, Clinical Oral Implants Research, International Journal of Oral \& Maxillofacial Implants, European Journal of Oral Implantology, Implant Dentistry, International Journal of Periodontics and Restorative Dentistry, International Journal of Prosthodontics, Journal of Prosthetic Dentistry and Clinical Implant Dentistry and Related Research. Además, se complementó esta fase con la búsqueda en la literatura gris: www.opengrey.eu.

Selección de artículos y extracción de datos. Se analizó los títulos, resúmenes y textos completos de los artículos que fueron registrados y se vaciaron los datos a cargo de dos revisores autónomos (E. R. y Y.C). Si hubo desacuerdo entre los revisores se resolvió mediante la intervención de un tercer revisor (M.A.). Para graduar la confiabilidad entre los verificadores, se midió las concordancias mediante el coeficiente Kappa de Cohen (K: 0.8).

Evaluación del riesgo de sesgo. Se evaluó el riesgo de sesgo de los ECA siguiendo el manual de Cochrane para intervenciones de revisiones sistemáticas Versión 5.1.0. (Higgins \& Green, 2011). Se analizó seis ítems del riesgo de sesgo: generación de la secuencia, ocultamiento de la información, cegamiento de los participantes y del personal, 
cegamiento de los evaluadores de los resultados, datos de resultados incompletos y notificación selectiva de los resultados, estos ítems se catalogaron como bajo (verde), no claro (amarillo) y alto riesgo de sesgo (rojo). Para la evaluación del riesgo de sesgo de ECC se utilizó la escala de Newcastle Ottawa (Lo et al.), que evaluó tres categorías (selección, comparabilidad y resultados) y nueve asignaciones (mayor a siete se consideró bajo riesgo de sesgo).

\section{RESULTADOS}

La búsqueda de la literatura, por los revisores autónomos (E.R. y Y.C.) encontraron un total de 930 artículos, de los cuales 922 fueron descartados después de la evaluación de títulos y resúmenes, a continuación, se examinaron el texto completo de 08 artículos, por último, un total de 04 estudios cumplieron los criterios de inclusión y se analizaron en la presente revisión sistemática, 03 ECA y 01 ECC, el diagrama de flujo se presenta en la Figura 1. La evaluación del riesgo de sesgo de los ECA se mues- tra en la Figura 2. De acuerdo a las sugerencias de Higgins \& Green, los ECA fueron de bajo riesgo de sesgo (Amin et al., 2018; Rojo et al., 2018, 2020) destacando que los ensayos clínicos de Rojo et al. $(2018,2020)$ cumplieron todos los ítems. La evaluación de riesgo de sesgo del ECC de Dellavia et al. (2014) según la escala Newcastle Ottawa obtuvo ocho asignaciones positivas de las tres categorías evaluadas, considerándose de bajo riesgo de sesgo (Tabla I).

Se realizaron un total de $87 \mathrm{CP}$ alrededor de piezas e implantes dentales, de las cuales, 45 cirugías fueron realizadas con ITB del AT y 42 cirugías con ITB del ALP. Dentro de las CP se ejecutaron 47 cirugías con la técnica de AVTB, 20 cirugías de aumento de tejido queratinizado y 20 cirugías de recubrimiento de recesiones gingivales. Se obtuvo un total de 67 ITC y 20 injertos de epitelio conectivo. Dos estudios utilizaron parámetros bidimensionales para evaluar los resultados, como la sonda periodontal (carolina del norte) y fotografías clínicas (Dellavia et al.; Amin et al.). Los artículos de Rojo et al. $(2018,2020)$ utilizaron el método tridimensional mediante un escáner óptico intraoral.

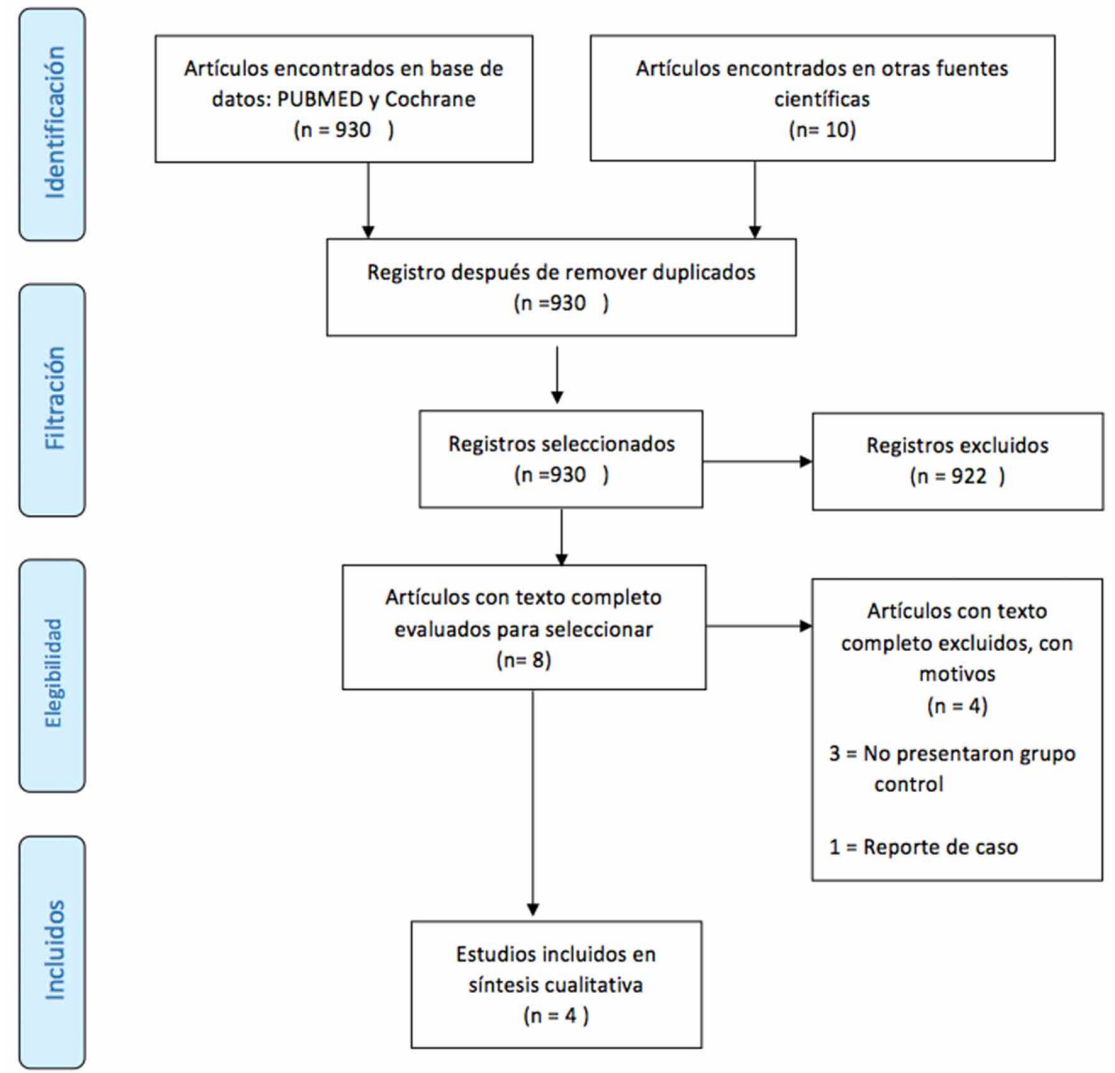

Fig. 1. Diagrama de flujo (formato PRISMA) filtración y proceso de elección.
A nivel de los resultados clínicos finales, en todos los ensayos clínicos no se encontraron diferencias estadísticamente significativas entre ambos grupos (Dellavia et al.; Amin et al.; Rojo et al., 2018, 2020). Pero se evidenció una diferencia estadísticamente significativa a favor del ITB del AT a nivel del parámetro clínico espesor gingival (Rojo et al., 2018; Amin et al.) y ancho de tejido queratinizado (Rojo et al., 2018, 2020), de igual manera Dellavia et al. demostró una diferencia clínica en los resultados a favor del ITC del AT.

Amin et al. evaluó la escala del dolor y obtuvo una diferencia estadísticamente significativa en las 2 primeras semanas a favor del grupo que obtuvo el ITB del AT. Se efectuó el seguimiento de los resultados desde las 8 semanas hasta los 12 meses (Tabla II). 
A
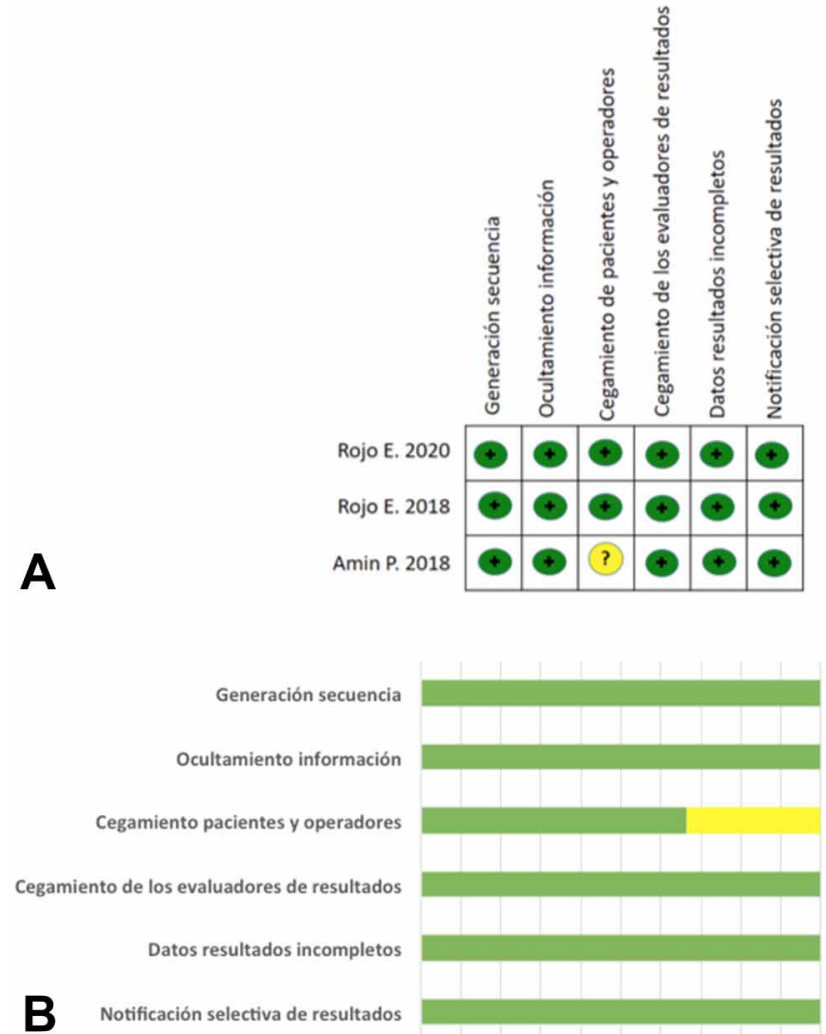

Fig. 2. Evaluación de los riesgos de sesgo según manual Cochrane, Versión 5.1.0, (a) resumen riesgo de sesgo de los ensayos clínicos aleatorizados incluidos (b) bajo, no claro, alto riesgo de sesgo.

\section{DISCUSIÓN}

Calidad de la evidencia y sesgos potenciales en el proceso de revisión. En la presente revisión sistemática de acuerdo a las descripciones de Higgins \& Green y la evaluación mediante la escala Newcastle Ottawa (Lo et al., 2014), los estudios incluidos (Dellavia et al.; Amin et al.; Rojo et al., 2018, 2020) mostraron un bajo riesgo de sesgo, lo que pone en evidencia que las metodologías empleadas en estos estudios son reproducibles y presentan bastante credibilidad. Es necesa- rio mencionar la variabilidad en las herramientas de medición de los resultados, esta variabilidad corresponde a la técnica quirúrgica realizada, para el recubrimiento de recesiones gingivales y el aumento de tejido queratinizado es ideal el empleo de herramientas bidimensionales como la fotografía clínica y la sonda periodontal (carolina del norte) y para cirugías de aumento de volumen se empleó las herramientas de medición tridimensionales como el escáner óptico intraoral, esta herramienta muestra una alta precisión, variando de 3 a $200 \mu \mathrm{m}$ y desde $0.05 \%$ a $1.5 \%$ en volumen, este sistema tridimensional representa el futuro de las mediciones en la cirugía de aumento de volumen (Marzadori et al., 2018). A pesar de la variabilidad en las herramientas de medición, no se encontraron diferencias estadísticamente significativas, tanto para los estudios que emplearon instrumentos bidimensionales y tridimensionales.

Hallazgos comparados con otros estudios. Estudios histológicos mostraron, que el contenido de colágeno en la lámina propia del tejido conectivo subepitelial del ALP y el AT son similares, con presencia de colágeno tipo 1 y 3 , no encontrando diferencias estadísticamente significativas entre ellas (Harris et al., 2003; Dellavia et al.; Sanz-Martin et al., 2019), estas evaluaciones histomorfométricas concuerdan con la presente revisión sistemática donde los resultados clínicos son similares con injertos de ambos sitios donantes. Asimismo, en los estudios incluidos utilizaron la técnica del injerto de "epitelio-conectivo desepitelizado", en la que se realizó la desepitelización de manera externa (excepto para la cirugía de aumento de tejido queratinizado), con esta técnica se obtiene el ITC superficial, adyacente al epitelio, que es más denso, estable y está sujeto a menor contracción/reabsorción durante la cicatrización, con respecto al que está próximo al periostio (Zuhr et al., 2014; Zucchelli \& Mounssif).

Con respecto a la evaluación del fenotipo gingival (espesor gingival y anchode tejido querati-nizado) de los sitios receptores en los procedimientos de cirugía periodontal, es un consenso que la presencia de un te-

Tabla I. Evaluación riesgo de sesgo, escala NEWCASTLE-OTTAWA para ensayos clínicos controlados (ECC).

\begin{tabular}{|c|c|c|c|c|c|c|c|c|c|c|c|c|}
\hline \multirow[t]{3}{*}{ AUTOR } & \multirow{3}{*}{$\begin{array}{l}\text { DISEÑO } \\
\text { DE } \\
\text { ESTUDIO }\end{array}$} & \multicolumn{9}{|c|}{$\begin{array}{c}\text { EVALUACIÓN DE RIESGO DE SESGO, ESCALA } \\
\text { NEWCASTLE - OTTAWA }\end{array}$} & \multirow{3}{*}{$\begin{array}{c}\text { TOTA } \\
L\end{array}$} & \multirow{3}{*}{ CONCLUSIÓN } \\
\hline & & \multicolumn{4}{|c|}{ SELECCIÓN } & \multicolumn{2}{|c|}{ COMPARABILIDAD } & \multicolumn{3}{|c|}{ RESULTADOS } & & \\
\hline & & 1 & 2 & 3 & 4 & 1 & 2 & 1 & 2 & 3 & & \\
\hline $\begin{array}{l}\text { Dellavia } \\
\text { C. } 2014\end{array}$ & ECC & & & & & & & & & & 8 & $\begin{array}{c}\text { BAJO } \\
\text { RIESGO } \\
\text { SESGO }\end{array}$ \\
\hline
\end{tabular}


Tabla II. Formato PICO (P: población, I: intervención, C: comparación, O: resultados)

\begin{tabular}{|c|c|c|c|c|c|c|c|}
\hline AUTOR & DISEÑO & POBLACIÓN & INTERVENCIÖN & COMPARACIÓN & RESULTADOS & & SEGUIMIENTO \\
\hline Rojo E. & ECA; & I: $16 \mathrm{ld}$ & AVTB $\infty n$ & AVTB con & Espesor tejido blando promedio & $\mathrm{p}=0,87$ & 12 meses \\
\hline \multirow[t]{5}{*}{2020} & paralelos & C: $15 \mathrm{Id}$ & ITC tuberosidad & ITC paladar & I: $0,04( \pm 0,23) \mathrm{mm} ; \mathbf{C}: 0,03( \pm 0,22) \mathrm{mm}$ & & \\
\hline & & & & & Ancho tejido queratinizado & $\mathrm{p}=\mathbf{0 , 0 2}$ & \\
\hline & & & & & \multirow{2}{*}{$\begin{array}{l}\text { I: } 0( \pm 0,32) \mathrm{mm} ; \quad \text { C: }-0,3( \pm 0,33) \mathrm{mm} \\
\text { Indice estético rosado }\end{array}$} & & \\
\hline & & & & & & $\mathrm{p}=0,59$ & \\
\hline & & & & & $\begin{array}{l}\text { I: } 8,54( \pm 2,43) \quad \text { C: } 8,37( \pm 2,46)\end{array}$ & & \\
\hline \multirow[t]{8}{*}{$\begin{array}{l}\text { Rojo E. } \\
2018\end{array}$} & $\begin{array}{l}\text { ECA; } \\
\text { grupos }\end{array}$ & I: $18 \mathrm{ld}$ & AVTB $\infty n$ & AVTB con & Espesor tejido blando promedio & $\mathrm{p}=0,64$ & \\
\hline & & & ITC tuberosidad & ITC paladar & \multirow{2}{*}{$\begin{array}{l}\text { I: } 0,79( \pm 1) \mathrm{mm} ; \quad \text { C: } 0,69( \pm 0,23) \mathrm{mm} \\
\text { Espesor tejido blando a } 6 \mathrm{~mm} \text { apical PC } \\
\text { I: } 0,81( \pm 0.33) \mathrm{mm} ; \mathbf{C}: 0,39( \pm 0,24) \mathrm{mm}\end{array}$} & & \\
\hline & & C: $15 \mathrm{Id}$ & & & & $\mathrm{p}=\mathbf{0 , 0 2}$ & \\
\hline & & & & & \multirow{2}{*}{ Espesor tejido blando a $7 \mathrm{~mm}$ apical $P C$} & $p=0,006$ & 3 meses \\
\hline & & & & & & & \\
\hline & & & & & Ancho tejido queratinizado & $\mathrm{p}=\mathbf{0 , 0 0 9}$ & \\
\hline & & & & & $\begin{array}{l}\text { I: } 0,83( \pm 0,61) \mathrm{mm} ; \mathbf{C}: 0,22( \pm 0,48) \mathrm{mm} \\
\text { Indice estético rosado }\end{array}$ & $\mathrm{p}=0,67$ & \\
\hline & & & & & I: $9,15( \pm 2,34) \quad$ C: $10,07( \pm 2,19)$ & & \\
\hline Amin $P$. & $\begin{array}{l}\text { ECA; boca } \\
\text { dividida }\end{array}$ & I: $20 \mathrm{Pd}$ & a) 10 IGL tuberosidad & a) 10 IGL paladar & AUMENTO TEJ. QUERATINIZADO & & \\
\hline \multirow[t]{11}{*}{2018} & & C: $20 \mathrm{Pd}$ & $\begin{array}{l}\text { b) } 10 \mathrm{CRC}+\mathrm{ITC} \\
\text { tuberosidad }\end{array}$ & $\begin{array}{l}\text { b) } 10 \mathrm{CRC}+\mathrm{ITC} \\
\text { paladar }\end{array}$ & Espesor gingival & $p=0,026$ & \\
\hline & & & & & \multirow{2}{*}{\multicolumn{3}{|c|}{$\begin{array}{l}\text { I: } 2,7( \pm 0,7) \mathrm{mm} ; \mathbf{C}: 2,1( \pm 0,7) \mathrm{mm} \\
\text { RECUBRIMIENTO RECESIONES GINGIVALES }\end{array}$}} \\
\hline & & & & & & & \\
\hline & & & & & \multirow{2}{*}{$\begin{array}{l}\text { Porcentaje recubrimiento } \\
\begin{array}{ll}\text { I: } 67( \pm 12) \% & \text { C: } 62( \pm 13) \%\end{array}\end{array}$} & $\mathrm{p}=0,102$ & \\
\hline & & & & & & & 2 meses \\
\hline & & & & & Espesor gingival & $\mathrm{p}=\mathbf{0 , 0 1 6}$ & \\
\hline & & & & & \multirow{2}{*}{$\begin{array}{l}\text { I: } 2,9( \pm 0,5) \mathrm{mm} ; \mathbf{C}: 2,3( \pm 0,6) \mathrm{mm} \\
\text { ESCALA DEL DOLOR }(1-10)\end{array}$} & & \\
\hline & & & & & & & \\
\hline & & & & & hasta 2 semanas & $\mathbf{p}<0,001$ & \\
\hline & & & & & \multirow{2}{*}{$\begin{array}{l}\text { I: } 2,6( \pm 2,2) \\
4 \text { a } 8 \text { semanas }\end{array}$} & & \\
\hline & & & & & & & \\
\hline Dellavia C. & ECC; grupo & I: $07 \mathrm{Pd}$ & AVTB $\infty n$ & AVTB con & \multicolumn{2}{|l|}{$\begin{array}{l}\text { I: } 0,0 \\
\text { Espesor aingival }\end{array}$} & \\
\hline 2014 & s paralelos & $\mathrm{C}: 07 \mathrm{Pd}$ & ITC tuberosidad & ITC paladar & Pre Qx $\quad$ I: $2,1 \pm 0,2 \mathrm{~mm} \quad$ C: $2,0 \pm 0,2 \mathrm{~mm}$ & & 12 meses \\
\hline
\end{tabular}

ECA: Ensayo clínico aleatorizado; ECC: Ensayo clínico controlado; C: control; I : intervención; Id: zona alrededor de implantes dentales; PC: pilar cicatrización; Pd: zona alrededor de piezas dentales; AVTB: Aumento de volumen de tejido blando; ITC: injerto tejido conectivo; IGL: injerto gingival libre; CRC: colgajo reposicionado coronalmente; Qx: quirúrgico.

jido gingival grueso promueve la resistencia al trauma, la recesión, la reducción de la inflamación clínica y la mejora predecible de los resultados quirúrgicos (Kim et al., 2020). En la presente revisión se encontró un mejor fenotipo gingival con ITB del AT (Dellavia et al.; Rojo et al., 2018; Amin et al.) y en las cirugías de AVTB en implantes se observó una mayor estabilidad del tejido queratinizado del área de la tuberosidad después de la colocación de la corona definitiva (Rojo et al., 2020), esto puede deberse a que, en evaluaciones histomorfométricas en la zona de la tuberosidad se evidenció un mayor porcentaje de lámina propia de 72,79 \% (menos propensa a la contracción post operatoria) y menor porcentaje de sub mucosa 4,89\% (tejido adiposo y glandular) a comparación del ALP donde se encontró un porcentaje en lámina propia de 51,08 \% y submucosa de $25,75 \%$ (más propensa a la contracción postoperatoria) (Sanz-Martin et al.), además, análisis moleculares mencionan que el colágeno de la zona de la tuberosidad es más susceptible a la reticulación (endurecimiento) por la presencia de una mayor relación LH2b/COL-1 (lisil hidroxilasa/Colágeno tipo 1) y menor probabilidad de degradarse por las MMPs (metaloproteinasas de matriz), Dellavia et al. sugiere que estas diferencias en la maduración del colágeno podría actuar como los principales determinantes en la respuesta hiperplásica del injerto del AT (Dellavia et al.).

En la revisión sistemática de Thoma et al., se demostró una mayor morbilidad asociada con los injertos de tejido blando autógeno en comparación con los sustitutos de tejido blando, en la presente revisión Amin et al. evaluó la escala del dolor del 1 al 10 y obtuvo una diferencia estadísticamente significativa a favor de las cirugías que obtuvieron ITB del AT en las dos primeras semanas post operatorias, en un reporte de caso de 90 pacientes Burkhardt et al. (2015) llegó a la conclusión que el grosor del injerto se correlacionó directamente con la cantidad de dolor percibido, sin embargo, la superficie desnuda de la herida no influyó en el nivel de dolor advertido por el paciente. Los resultados sobre la morbilidad del paciente son de gran relevancia y podrían ayudar en la elección del sitio donante del ITB hacia sustitutos de tejidos blandos en el futuro. 
Un factor importante a considerar al momento de valorar los procedimientos quirúrgicos es el tiempo de evaluación (seguimiento) para observar los resultados finales, al respecto Sculean et al. proporcionó una visión general sobre la biología y la cicatrización de las heridas de tejidos blandos alrededor de dientes e implantes dentales, donde llegó a la conclusión de que la cicatrización epitelial después de la terapia quirúrgica parece completarse después de un periodo de 7 a 14 días, a partir de los 14 días post quirúrgicos se logra una integridad estructural de una herida en maduración entre una superficie radicular desnuda y un colgajo de tejido blando. La formación del ancho biológico y la maduración en la función de barrera alrededor de los implantes transmucosos requiere de 6 a 8 semanas de cicatrización, Amin et al. evaluó los resultados a las 8 semanas, mientras que, en una revisión sistemática y meta-análisis de Lin et al. (2018) reveló que la estabilidad del tejido blando en términos de ancho de tejido queratinizado y recesión de la mucosa a nivel vestibular se obtiene después de los tres meses de la cirugía.

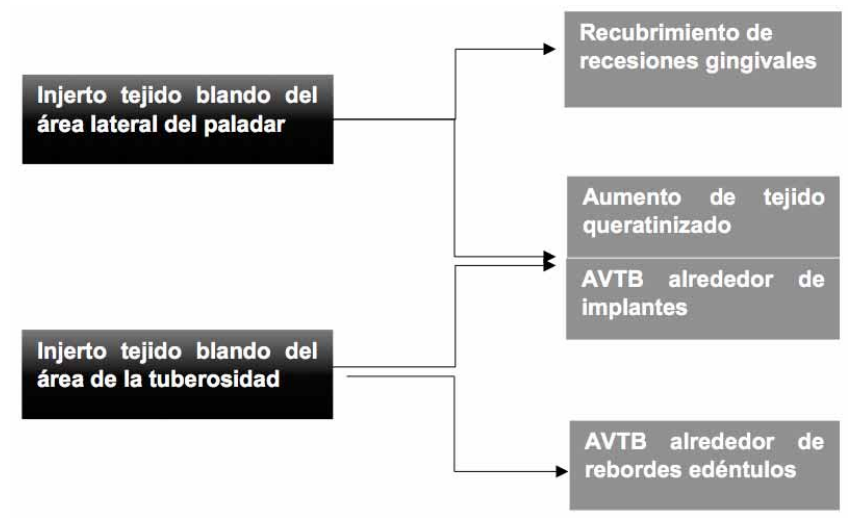

Fig. 3. Manejo clínico de diversos sitios donantes de injerto de tejido blando. AVTB: Aumento de volumen de tejido blando. TC: Tejido conectivo

ÁREA LATERAL DEL PALADAR

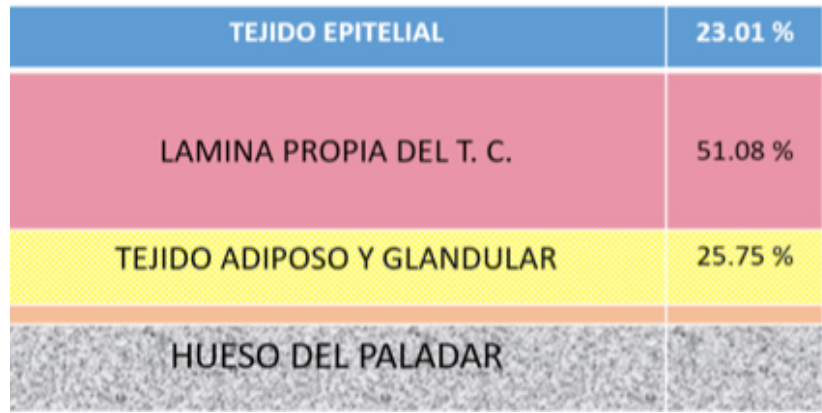

De acuerdo a la evidencia encontrada y a la experiencia clínica, la decisión de dónde extraer el injerto de tejido blando dependerá de la indicación para la cual se utilizará el injerto y de la cantidad de tejido disponible en los sitios donantes elegibles. En base a los resultados encontrados en la presente revisión, se propone el siguiente protocolo para los procedimientos de cirugía periodontal/periimplantar con injerto de tejido blando (Fig. 3).

El ALP estaría indicada para el recubrimiento de recesiones gingivales, aumento de tejido queratinizado y aumento de volumen en implantes dentales (Zuhr et al.) siendo ideal las zonas de elección el área palatina de premolares y primer molar maxilar a $2 \mathrm{~mm}$ del margen gingival (Rojo et al., 2018; Amin et al.). El AT podría estar indicada para las cirugías de aumento de tejido queratinizado y AVTB en rebordes edéntulos e implantes dentales facilitándose la toma si se tiene solo el 1er molar. En ambas situaciones se recomienda obtener el ITB con mayor componente histológico de lámina propia que esta adyacente al epitelio ortoqueratinizado, respetando las constantes anatómicas (Zuhr et al.; Sanz et al.) (Fig. 4).

Limitaciones de los artículos incluidos. Solamente cuatro estudios comparativos fueron encontrados en la presente revisión siendo importante tener más metodología de este tipo, de preferencia aleatorizada, para tomar decisiones clínicas válidas y predecibles a largo tiempo. Un ensayo clínico realizó una evaluación a corto plazo, se sugiere en próximos estudios prolongar el tiempo de evaluación de los resultados, para una mejor valoración en la estabilidad de los injertos de ambos sitios donantes. Asimismo, considerar que solamente un estudio evaluó la escala del dolor siendo muy importante los resultados reportados por el paciente, los cuales proporcionan información adicional con respecto a la morbilidad luego de la toma del injerto de tejido blando.

ÁREA TUBEROSIDAD

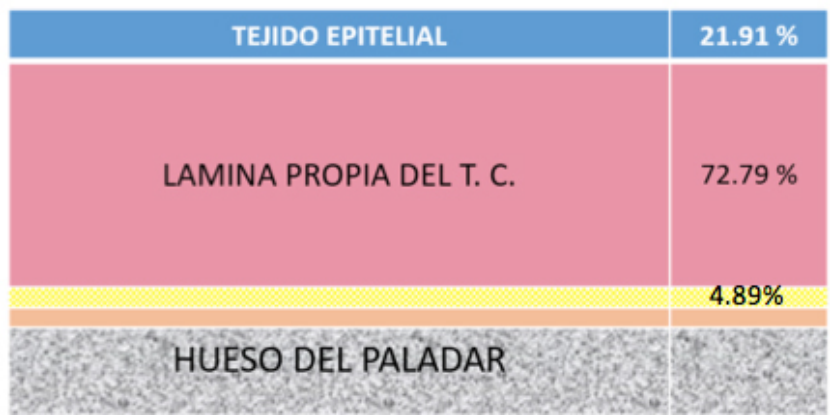

Fig. 4. Análisis histomorfométrico según Sanz-Martín et al. (2019). 


\section{CONCLUSIONES}

- Las zonas donadoras de injerto de tejido blando, tanto del área del paladar y de la tuberosidad brindan satisfactorios resultados clínicos similares a corto plazo para los procedimientos de cirugía plástica periodontal/periimplantar.

- El injerto de tejido blando del área de la tuberosidad mejora el fenotipo gingival del área receptora y brinda mayor estabilidad del tejido queratinizado, asimismo disminuye la morbilidad en cuanto a dolor postoperatorio en las primeras 2 semanas.

- El injerto de tejido conectivo superficial, adyacente al epitelio, es más denso, estable y está sujeto a menor contracción/reabsorción durante la cicatrización.

- La elección de la mejor zona donadora para elegir un injerto de tejido blando dependerá de la experiencia clínica y de la disponibilidad de tejido blando para los propósitos clínicos que se busquen.

RAMOS-PILCO, E.; CONDORI, S. Y. \& ALARCÓN, M. A. Palate or Tuberosity, which is the best donor site for a Soft tissue graft? A systematic review. Int. J. Odontostomat., 14(4):602-609, 2020.

ABSTRACT: The objective of the study was to compare, through a systematic review, the clinical results of periodontal / peri-implant plastic surgery $(\mathrm{CP})$ procedures with autogenous soft tissue graft (ITB) obtained from the lateral palate area (ALP) versus the tuberosity area (AT). We conducted an electronic search of clinical trials in the Medline/Pubmed, Cochrane database and the journals of Periodontics and Implants with the greatest impact according to the Web of Science, to find articles published until April 2020. The risk of bias of the articles added was assessed according to the Cochrane Manual Version 5.1.0 for randomized clinical trials and the Newcastle-Ottawa scale for controlled clinical trials. From the initial sample of 930 articles, four clinical trials were included (three randomized) in the present study, where a total of $87 \mathrm{PC}$ were performed around dental pieces and implants, of which 42 surgeries were performed with ITB of the ALP and 45 surgeries with ITB of the AT, the results were evaluated from the 8 weeks to 12 months. No statistically significant differences were found in the clinical results between the two groups, the gingival phenotype in the receiving area was improved for the group that obtained the ITB of the AT and the level of pain at the donor site was lower in the first two weeks than the ALP donor site. The studies showed a lowrisk of bias on average. Both soft tissue graft donor areas provide similar clinical results, grafting the tuberosity area improves the gingival phenotype of the recipient area and reduces post-operative pain of the donor site in the first few weeks.

KEY WORDS: dental implants, palate, connective tissue, autograft, gingival recession.

\section{REFERENCIAS BIBLIOGRÁFICAS}

Amin, P. N.; Bissada, N. F.; Ricchetti, P. A.; Silva, A. P. B. \& Demko, C. A. Tuberosity versus palatal donor sites for soft tissue grafting: A split-mouth clinical study. Quintessence Int., 49(7):589-98, 2018.

Burkhardt, R.; Hämmerle, C. H. F.; Lang, N. P. \& Research Group on Oral Soft Tissue Biology \& Wound Healing. Self-reported pain perception of patients after mucosal graft harvesting in the palatal area. J. Clin. Periodontol., 42(3):281-7, 2015.

Cairo, F.; Pagliaro, U. \& Nieri, M. Soft tissue management at implant sites. J. Clin. Periodontol., 35(8 Suppl.):163-7, 2008.

Dellavia, C.; Ricci, G.; Pettinari, L.; Allievi, C.; Grizzi, F. \& Gagliano, N. Human palatal and tuberosity mucosa as donor sites for ridge augmentation. Int. J. Periodontics Restorative Dent., 34(2):179-86, 2014.

Hämmerle, C. H. F. \& Tarnow, D. The etiology of hard- and softtissue deficiencies at dental implants: A narrative review. J. Periodontol., 89 Suppl. 1:S291-S303, 2018.

Harris, R. J. Histologic evaluation of connective tissue grafts in humans. Int. J. Periodontics Restorative Dent., 23(6):57583, 2003.

Higgins, J. P. T. \& Green, S. Cochrane Handbook for Systematic Reviews of Interventions. Versión 5.1.0 [updated March 2011]. Londres, The Cochrane Collaboration, 2011. Disponible en: https://handbook-5-1.cochrane.org/

Jung, R. E.; Herzog, M.; Wolleb, K.; Ramel, C. F.; Thoma, D. S. \& Hämmerle, C. H. F. A randomized controlled clinical trial comparing small buccal dehiscence defects around dental implants treated with guided bone regeneration or left for spontaneous healing. Clin. Oral Implants Res., 28(3):34854, 2017.

Kim, D. M.; Bassir, S. H. \& Nguyen, T.T. Effect of gingival phenotype on the maintenance of periodontal health: An American Academy of Periodontology best evidence review. J. Periodontol., 91(3):311-38, 2020.

Lin, C. Y.; Chen, Z.; Pan, W. L. \& Wang, H. L. Impact of timing on soft tissue augmentation during implant treatment: A systematic review and meta-analysis. Clin. Oral Implants Res., 29(5):508-21, 2018.

Lo, C. K. K.; Mertz, D. \& Loeb, M. Newcastle-Ottawa Scale: comparing reviewers' to authors' assessments. BMC Med. Res. Methodol., 14:45, 2014.

Marzadori, M.; Stefanini, M.; Mazzotti, C.; Ganz, S.; Sharma, P. \& Zucchelli, G. Soft-tissue augmentation procedures in edentulous esthetic areas. Periodontol. 2000, 77(1):111-22, 2018.

Moher, D.; Liberati, A.; Tetzlaff, J.; Altman, D. G. \& PRISMA Group. Preferred reporting items for systematic reviews and meta-analyses: the PRISMA statement. PLoS Med., 6(7):e1000097, 2009. 
Rojo, E.; Stroppa, G.; Sanz-Martin, I.; Gonzalez-Martín, O.; Alemany, A. S. \& Nart, J. Soft tissue volume gain around dental implants using autogenous subepithelial connective tissue grafts harvested from the lateral palate or tuberosity area. A randomized controlled clinical study. J. Clin. Periodontol., 45(4):495-503, 2018.

Rojo, E.; Stroppa, G.; Sanz-Martin, I.; Gonzalez-Martín, O. \& Nart, J. Soft tissue stability around dental implants after soft tissue grafting from the lateral palate or the tuberosity area - A randomized controlled clinical study. J. Clin. Periodontol., 47(7):892-9, 2020.

Sanz, M.; Simion, M. \& Working Group 3 of the European Workshop on Periodontology. Surgical techniques on periodontal plastic surgery and soft tissue regeneration: consensus report of Group 3 of the 10th European Workshop on Periodontology. J. Clin. Periodontol., 41 Suppl. 15:S927, 2014

Sanz-Martín, I.; Rojo, E.; Maldonado, E.; Stroppa, G.; Nart, J. \& Sanz, M. Structural and histological differences between connective tissue grafts harvested from the lateral palatal mucosa or from the tuberosity area. Clin. Oral Investig., 23(2):957-64, 2019

Schwarz, F.; Sahm, N. \& Becker, J. Impact of the outcome of guided bone regeneration in dehiscence-type defects on the long-term stability of peri-implant health: clinical observations at 4 years. Clin. Oral Implants Res., 23(2):191-6, 2012.

Sculean, A.; Gruber, R. \& Bosshardt, D. D. Soft tissue wound healing around teeth and dental implants. J. Clin. Periodontol., 41 Suppl. 15:S6-22, 2014.

Thoma, D. S.; Buranawat, B.; Hämmerle, C. H. F.; Held, U. \& Jung, R. E. Efficacy of soft tissue augmentation around dental implants and in partially edentulous areas: a systematic review. J. Clin. Periodontol., 41 Suppl. 15:S77-91, 2014.

Zucchelli, G. \& Mounssif, I. Periodontal plastic surgery. Periodontol., 68(1):333-68, 2015.

Zuhr, O.; Bäumer, D. \& Hürzeler, M. The addition of soft tissue replacement grafts in plastic periodontal and implant surgery: critical elements in design and execution. J. Clin. Periodontol., 41 Suppl. 15:S123-42, 2014.
Dirección para correspondencia:

Ramos Pilco, Edwin Pascual

Tacna

PERÚ

Email: edy4208@gmail.com

Recibido : 27-04-2020

Aceptado: 24-06-2020 